\title{
Arrival Time Difference Estimation of Ultrasonic Signals from Partial Discharge in Electric Power Equipments
}

\author{
Jinxi Hu, Wenhong Liu*, Haotian Zhang, Hang Liu, Keni Xu, Mianmian Wang \\ School of Electronic Information, Shanghai Dianji University, Shanghai, China \\ Email address: \\ 602734011@qq.com (Jinxi Hu), liuwenhong@sdju.edu.cn (Wenhong Liu) \\ ${ }^{*}$ Corresponding author \\ To cite this article: \\ Jinxi Hu, Wenhong Liu, Haotian Zhang, Hang Liu, Keni Xu, Mianmian Wang. Arrival Time Difference Estimation of Ultrasonic Signals from \\ Partial Discharge in Electric Power Equipments. Journal of Electrical and Electronic Engineering. Vol. 7, No. 5, 2019 , pp. 113-119. \\ doi: $10.11648 /$ j.jeee.20190705.13
}

Received: October 12, 2019; Accepted: October 29, 2019; Published: November 8, 2019

\begin{abstract}
Partial discharge of power equipment is one of the common faults in power systems. How to quickly and accurately determine the location of partial discharge is a problem that needs to be solved in practice. The signal arrival time difference estimation technique in signal processing is one of the effective methods to solve this problem. When the power equipment is partially discharged, an ultrasonic signal is generated. Therefore, the local discharge can be positioned according to the ultrasonic signal, however, the traditional signal arrival time difference estimation methods are not ideal for the actual low signal-to-noise ratio and narrow-band ultrasonic signals. In this paper, an improved correlation coefficient waveform comparison time difference estimation algorithm based on complete ensemble empirical mode decomposition with adaptive noise (CEEMDAN), threshold denoising is proposed, referred to as CEEMDAN-TDE. Firstly, according to the characteristics of the actual ultrasonic signals, the double-exponential decay oscillation model is used to model the partial discharge ultrasonic signals, and Gaussian white noises are added as the interference signals. secondly, the CEEMDAN threshold denoising is used to improve the signal-to-noise ratio of the partial discharge signals; thirdly, the cross-correlation coefficient is calculated, then the arrival time difference can be obtained by comparing the waveforms of the correlation coefficients, and the partial discharge location information is known. The computer simulations of the CEEMDAN-TDE method, and the generalized correlation method, LMS method, and correlation coefficient waveform comparison method estimation are performed. Experimental results show that the estimating performance in arrival time difference of proposed method, CEEMDAN-TDE, is better than the other three methods' under low SNR and narrowband. The CEEMDAN-TDE method has the hopeful more application in practice.
\end{abstract}

Keywords: Partial Discharge, Ultrasonic Signals, Arrival Time Difference Estimation, Low SNR, Narrowband Signals

\section{Introduction}

Partial discharge (PD), as a key feature of high-voltage electrical insulation aging, its immediate detection is of great significance for the safe operation of power systems [1]. Ultrasonic signals are generated during partial discharge, which can be used to locate the PD signal. The ultrasonic localization method is a kind of charged detection and positioning method with strong anti-electromagnetic interference, non-intrusive and low cost. extensive attention $[2,3]$.

In various spatial localization algorithms based on the arrival time difference of the signal source, the accuracy of the time difference estimation is one of the key factors for the final positioning accuracy. The existing time difference estimation methods mainly include correlation analysis method, phase spectrum estimation method and adaptive time delay estimation method [4-10]. The implementation of the traditional time difference estimation algorithm is mostly based on the assumption that the estimated signal is a stationary, wideband signal. However, the PD ultrasonic signals actually collected at the scene usually appear as non-stationary, non-linear narrow-band signals with attenuated oscillations. According to the nature of the Fourier transform, the narrower the frequency domain of the signal 
and the wider the time domain, most of the peak detection algorithms are affected by the time domain broadening and the peak is not obvious. Moreover, the collected signals in the field usually contain interference signals such as white noise and periodic narrow-band interference, which causes the accuracy of the traditional time difference estimation algorithm to be seriously reduced or even invalid.

The literature avoids the peak detection problem by using the correlation coefficient waveform comparison method, and improves the accuracy of the narrow-band signal time difference estimation, but its estimation accuracy is significantly reduced in the case of low SNR [11]. Based on the literature, the waveform comparison time difference estimation method based on wavelet transform is applied to the passive location of radio signals, which improves its robustness under low SNR conditions [12]. However, the denoising performance of wavelet transform is determined by the wavelet base selected by humans in advance, which has the disadvantages of subjectivity and non-adaptation. Empirical Mode Decomposition, referred to as EMD, Denoising is a data-driven, adaptive denoising method that does not require a basis function. The literature proves that the EMD noise reduction effect is better than wavelet denoising for PD signals, but There are disadvantages that easily cause modal aliasing [13]. Huang proposed the overall empirical mode decomposition, referred to as EEMD, which eliminates the modal aliasing phenomenon by adding Gaussian white noise, but the noise is difficult to completely eliminate and the signal reconstruction error is large. Torres proposed Complete Ensemble Empirical Mode Decomposition with Adaptive Noise, referred to as CEEMDAN, by adaptively adding Gaussian white noise after EMD decomposition, reducing residual noise and greatly reducing signal reconstruction error [14]. In this paper, an improved waveform comparison time difference estimation algorithm, referred to as CEEMDAN-TDE, based on CEEMDAN denoising is applied to the time difference estimation of PD ultrasonic signals.

\section{Improved Waveform time Difference Estimation Algorithm Based on CEEMDAN}

\subsection{CEEMDAN Basic Principles}

CEEMDAN is based on the improvement of the EMD principle. EMD adaptively decomposes the signal into multiple intrinsic mode functions (IMF), but it produces modal aliasing effects, which makes some IMFs meaningless. CEEMDAN solves this problem by adaptively adding Gaussian white noise in the IMF. The research proves that the method effectively weakens the modal aliasing problem and the reconstruction error is negligible. The calculation steps are as follows $[15,16]$ :

1) Adding a positive-negative pair of zero-mean unit variance white noise with a magnitude equal to a in the original signal $\mathrm{s}(\mathrm{t})$ :

$$
s^{\prime}(t)=s(t)+(-1)^{q} a n^{i}(t)
$$

By performing $\mathrm{N}$ EMD decompositions on $s^{\prime}(t), \mathrm{N}$ first-order component $I M F_{1}^{i}(t)$ and residual residuals $r_{1}^{i}(t)$ are obtained:

$$
s^{\prime}(t)=I M F_{1}^{i}(t)+r_{1}^{i}(t)
$$

Then, the integrated mean is obtained for $\mathrm{N} I M F_{1}^{i}(t)$, and the final first-order component $\overline{I M F_{1}(t)}$ is obtained:

$$
\overline{I M F_{1}(t)}=\frac{1}{N} \sum_{1}^{i} I M F_{1}^{i}(t)
$$

Easy to get the final first residual:

$$
r_{1}(t)=s(t)-\overline{I M F_{1}(t)}
$$

Adding the zero-mean unit variance white noise of the same magnitude to the positive and negative pairs of EMD decomposition in $r_{1}(t)$ and decomposing it again for $\mathrm{N}$ times EMD to obtain the second order component $I M F_{2}^{i}(t)$ and the residual residual $r_{2}^{i}(t)$ :

$$
r_{1}(t)+(-1)^{q} a E_{1}\left(n^{i}(t)\right)=I M F_{2}^{i}(t)+r_{2}^{i}(t)
$$

Find the integrated mean for $\mathrm{N} I M F_{2}^{i}(t)$ to get the final second-order component $\overline{I M F_{2}(t)}$ :

$$
\overline{I M F_{2}(t)}=\frac{1}{N} \sum_{1}^{i} I M F_{2}^{i}(t)
$$

The same second residual:

$$
r_{2}(t)=r_{1}(t)-\overline{I M F_{2}(t)}
$$

Repeat the above steps $\mathrm{m}$ times until the signal cannot continue to decompose, and the component of the m-stage order and the final residual $\mathrm{R}(\mathrm{t})$ can be obtained. The original signal can be expressed as:

$$
s(t)=\sum_{m=1}^{m} \overline{I M F_{m}(t)}+R(t)
$$

\subsection{CEEMDAN Denoising Principle}

Generally speaking, after the noise signal is decomposed by CEEMDAN, the noise is mainly concentrated in the high frequency IMF component. Therefore, the simplest method of denoising is to directly remove the high frequency IMF component and reconstruct the remaining IMF component to obtain the denoising signal. The boundary between high and low frequencies is usually determined using the correlation coefficient method. However, the high-frequency part is not completely noise, and the useful signal is also discarded, resulting in failure to achieve the desired denoising effect.

Wavelet threshold denoising is a common denoising method that preserves the noise signal at certain scales while preserving the useful signal by setting certain conditions. The research proves that, the joint denoising method combining CEEMDAN and wavelet threshold denoising is better than the single method [16]. The flow chart is shown in Figure 1. 
$I M F_{1-k}$ is the component that needs to be denoised by wavelet threshold, and $I M F_{k-n}$ is which didn't., $\mathrm{k}$ is determined by the correlation coefficient method.

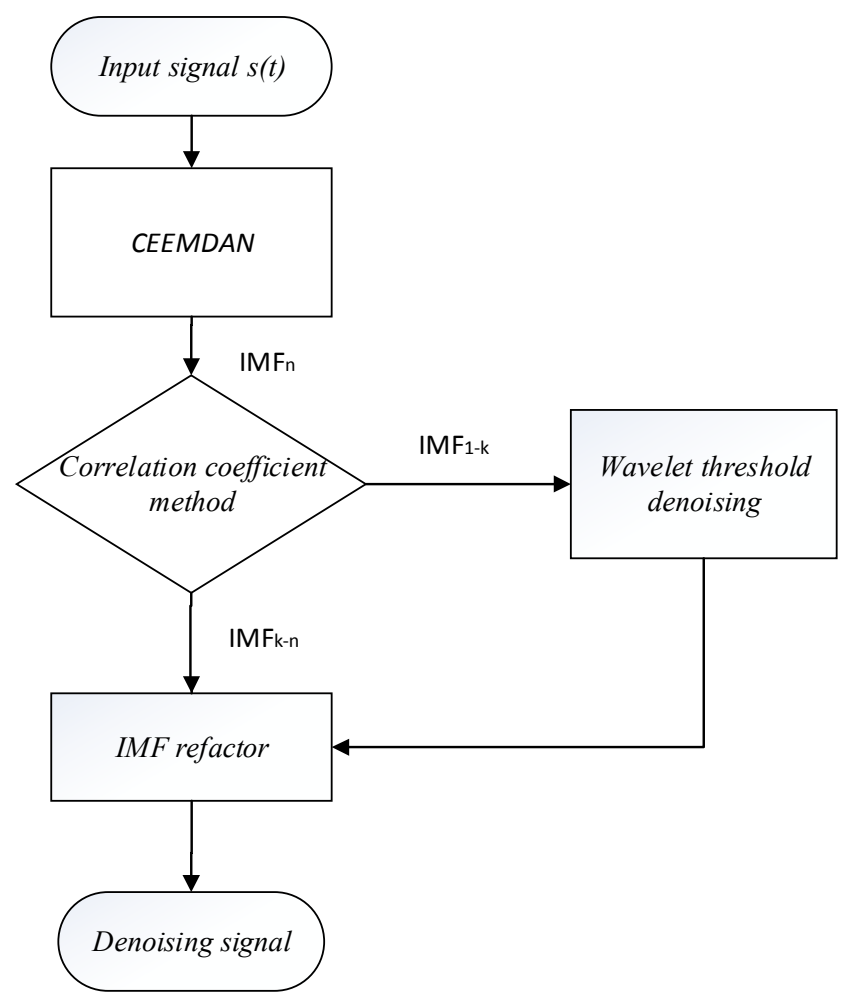

Figure 1. CEEMDAN threshold denoising algorithm flow chart.

\subsection{Correlation Coefficient Waveform Comparison Principle}

The correlation coefficient waveform comparison method converts the time difference information of the source signal into its correlation function, and obtains the time difference information by comparing the autocorrelation function of one signal and the waveform of the cross correlation function of the two signals [11].

Set up two-way signal model:

$$
\left\{\begin{array}{c}
s_{1}(t)=x(t)+n_{1}(t) \\
s_{2}(t)=x(t-D)+n_{2}(t)
\end{array}\right.
$$

In the above formula, $x(t)$ and $x(t-D)$ are input signals, and $D$ is the time difference between the two signals. $n_{1}(t)$ and $n_{2}(t)$ are mutually independent Gaussian white noises.

According to the nature of the correlation function, make $x(t)=x_{1}, \mathrm{R}(\cdot)$ Is seeking correlation. It can be seen that $R_{s_{1} s_{1}}(m)=R_{x_{1} x_{1}}(m), R_{s_{1} s_{1}}(m)=R_{x_{1} x_{1}}(m-D)$, Then the time difference between the two signals is transferred to the correlation function [8]. In practice, accurate correlation function values cannot be obtained, and are generally expressed by their estimated values:

$$
\left\{\begin{array}{l}
R_{S_{1} S_{1}}(m)=\frac{1}{N-m} \sum_{n=0}^{N-1-m} s_{1}(n) s_{1}(n+m) \\
R_{S_{1} S_{2}}(m)=\frac{1}{N-m} \sum_{n=0}^{N-1-m} s_{1}(n) s_{2}(n+m)
\end{array}\right.
$$

$\mathrm{N}$ is the number of signal sampling points, and the time difference can be obtained by comparing the estimated waveforms. This avoids the effect of the narrowband signal's ductility in the time domain on peak detection. The specific calculation steps are:

1) According to formula (10), calculate $R_{s_{1} s_{1}}(m)$, $R_{s_{1} s_{2}}(m)$.

2) Take a waveform of equal length from each of $\hat{R}_{s_{1} s_{1}}(m)$ and $\hat{R}_{s_{1} s_{2}}(m)$, find the correlation coefficient, and select the two waveforms with the largest correlation coefficient for comparison. The relative position on the time axis is the corresponding time difference estimation value.

3) Repeat step 2), perform histogram statistics on the obtained multiple estimated values, and select the value with the most repetition times as the final result.

\subsection{Waveform Comparison Method Based on CEEMDAN Improvement}

Based on the literature, the combination of wavelet transform and waveform comparison method improves the time difference estimation accuracy of wireless RF narrow-band signals at low SNR [12]. However, wavelet transform requires artificial selection of wavelet base and does not have adaptability. In the literature, EMD wavelet denoising and bi-spectrum estimation are combined to estimate the time difference of the local high-frequency signal [17]. However, the bi-spectrum estimation algorithm still uses peak detection, and the calculation amount is large, and the EMD itself also has problems such as modal aliasing. The CEEMDAN-based waveform comparison time difference estimation algorithm proposed in this paper aims to improve the time difference estimation accuracy of PD ultrasonic narrow-band signals in power equipment in complex environments. The flow chart is as follows:

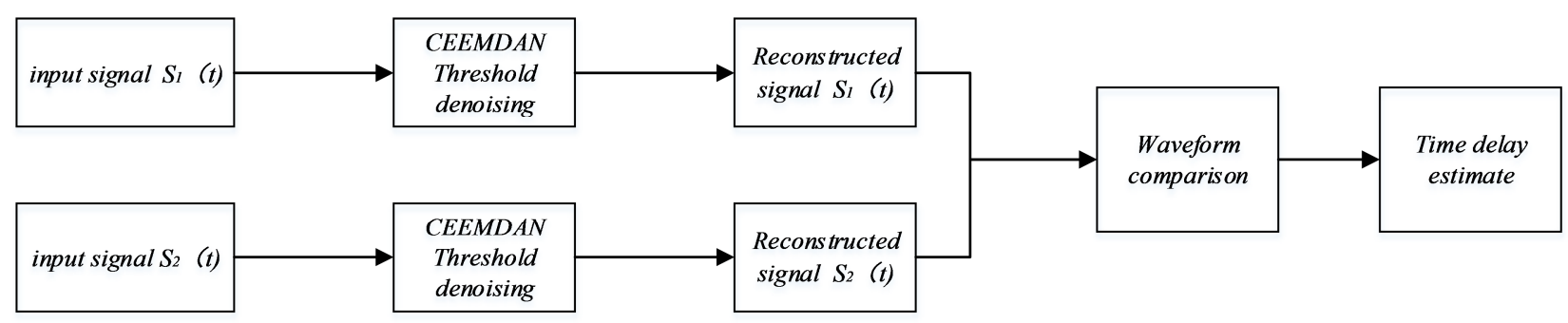

Figure 2. Schematic diagram of waveform comparison method based on CEEMDAN. 


\section{Simulation Research}

\subsection{PD Simulated Signal}

The PD simulation signal is usually represented by a single exponential decay pulse, a single exponential decay oscillation pulse model, a double exponential oscillation pulse, and a double exponential decay oscillation pulse model [14]. Double exponential decay oscillation pulse model

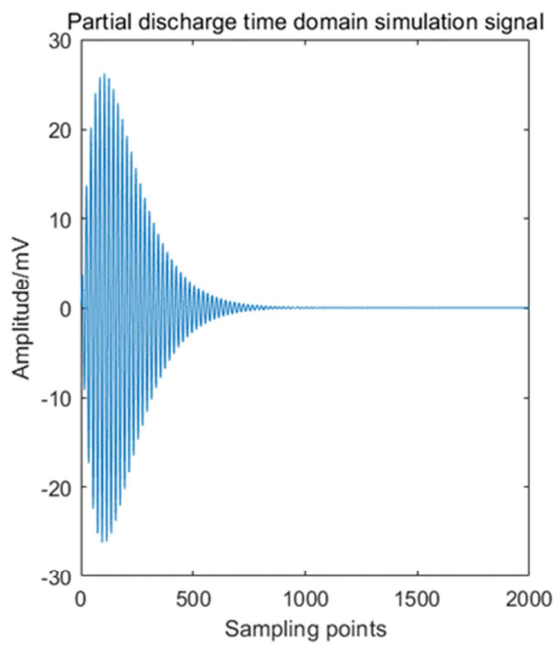

(a)PD time domain simulation signal

$$
\mathrm{S}(\mathrm{t})=\mathrm{A}\left(e^{-\frac{1.3 t}{\tau}}-e^{-\frac{1.3 t}{\tau}}\right) \sin \left(2 \pi f_{c} t\right)
$$

In the above formula, $A$ is the partial amplitude of the PD signal, $\tau$ is the attenuation constant, and $f_{c}$ is the oscillation constant. $A=300 \mathrm{mv}, f_{c}=500 \mathrm{kHz}, \tau=2.5 \mu \mathrm{s}$. The number of sampling points is $N=2000$, and the sampling frequency is $10 M$. The time domain diagram and the frequency domain diagram are shown in Figure 3.

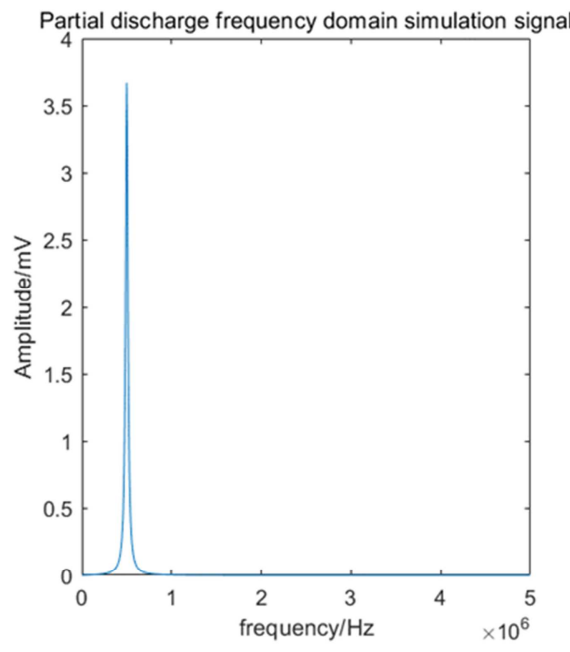

(b)PD frequency domain simulation signal

Figure 3. PD ultrasound simulation signal time domain and frequency domain diagram.

Using the two-way signal model in equation (9), assume that the ultrasonic sensor installation positions are point $\mathrm{A}$ and point $\mathrm{B}$, respectively, $\mathrm{D}=100$, and the instantaneous difference is $10 \mu \mathrm{s}$. Narrowband interference is usually different from the frequency band of the actual PD ultrasonic signal, and can be directly filtered by a band-pass filter [19]. Therefore, this paper mainly studies broadband white noise interference.
Gaussian white noise with a signal-to-noise ratio of $-15 \mathrm{~dB}$ is added separately. The original signal and the noise-added signal are as shown in the figure. The PD ultrasonic signal is basically submerged in the noise, and the time difference of the two signals cannot be directly observed by the waveform comparison method.

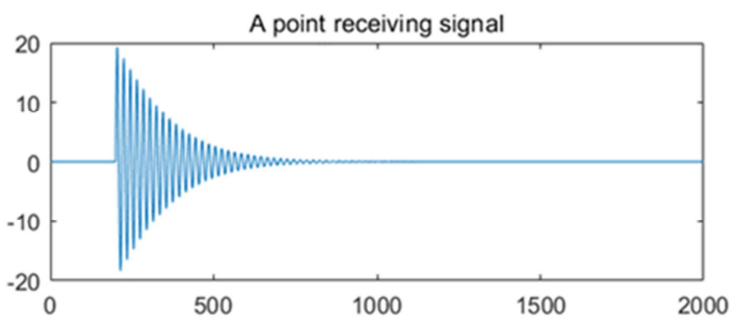

(a)A point receiving signal

B point receiving signal

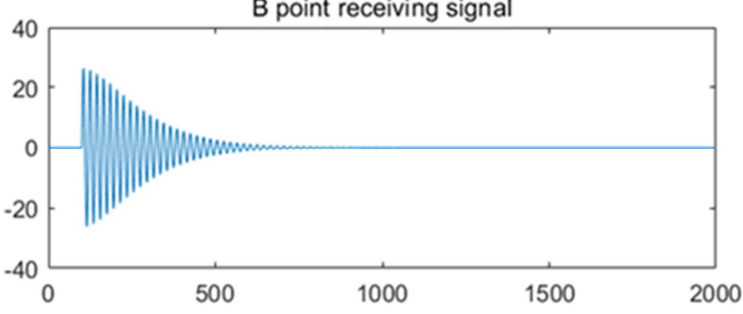

(c) B point receiving signal

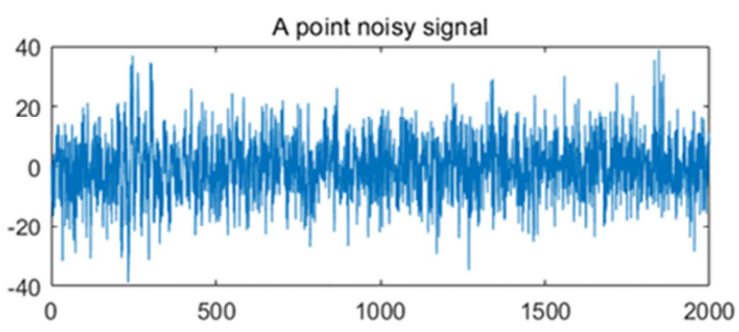

(b) A point noisy signal

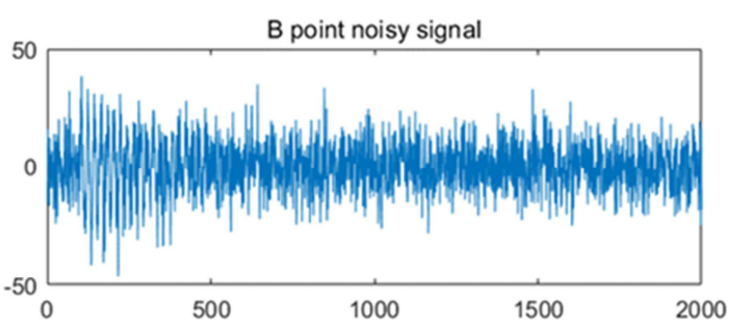

(d) B point noisy signal

Figure 4. Source and noisy signals at points $A$ and $B$. 


\subsection{CEEMDAN Denoising Signal}

The EMD threshold denoising is performed on the PD ultrasonic simulation signal at point $\mathrm{A}$, and point $\mathrm{B}$ is the same.
Figure 5 is a modal component obtained by CEEMDAN decomposition of the A-point PD ultrasonic signal.

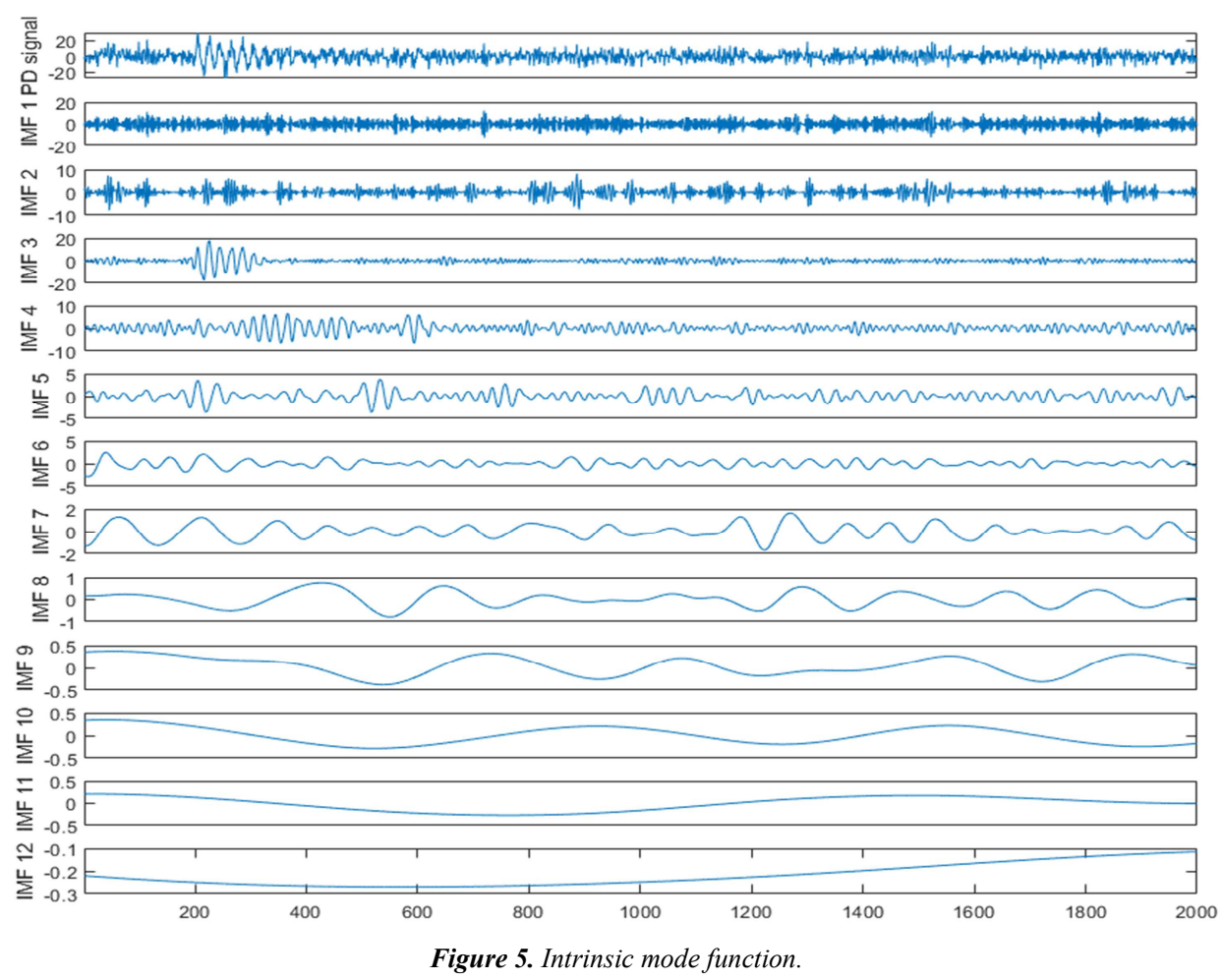

The white noise autocorrelation takes the maximum value at zero [16]. Gaussian noise mainly exists in $I M F_{1} \sim I M F_{5}$ through autocorrelation analysis of each IMF. Therefore, the wavelet threshold denoising is performed on the first five

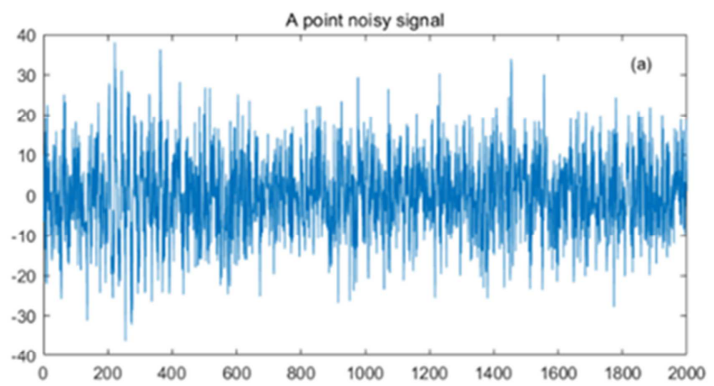

a. A point noisy signal

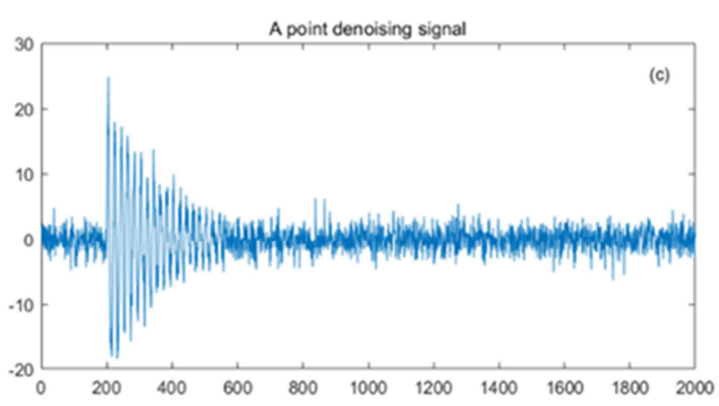

c. A point denoising signal modal components, and threshold selection is the same as the literature [12]. After the pair and the remaining IMF are reconstructed, the obtained denoising signal is as shown in Figure 6.

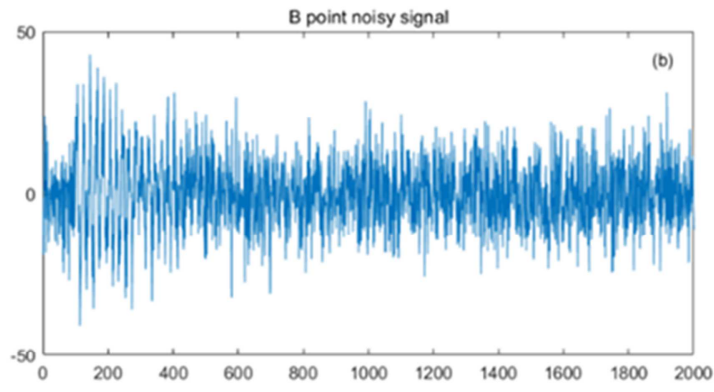

b. B point noisy signal

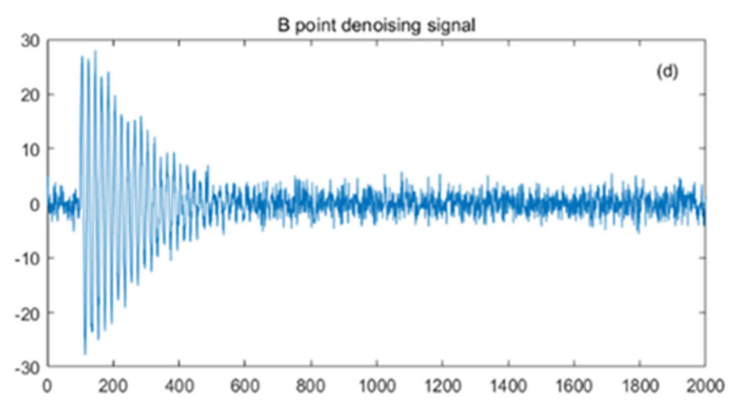

d. B point denoising signal

Figure 6. CEEMDAN threshold denoising signal. 
From the figure, most of the Gaussian noise is filtered out by the CEEMDAN threshold denoising process, and the signal-to-noise ratio is significantly improved, and the PD signal is revealed. However, the residual Gaussian noise causes the starting point of the PD signal to be still difficult to determine. According to equation (10), the autocorrelation

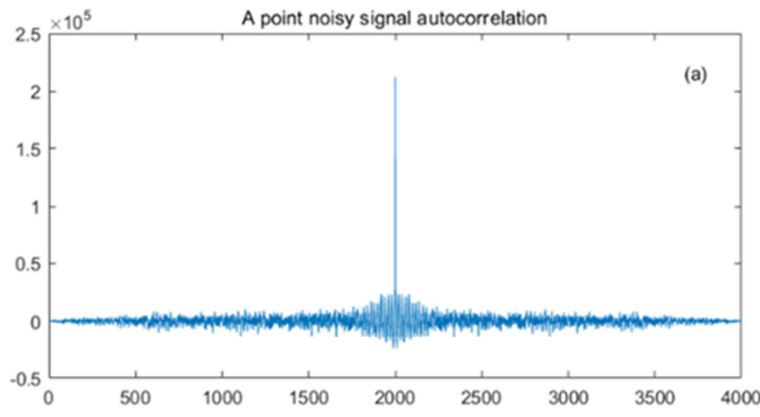

a. A point noisy signal autocorrelation

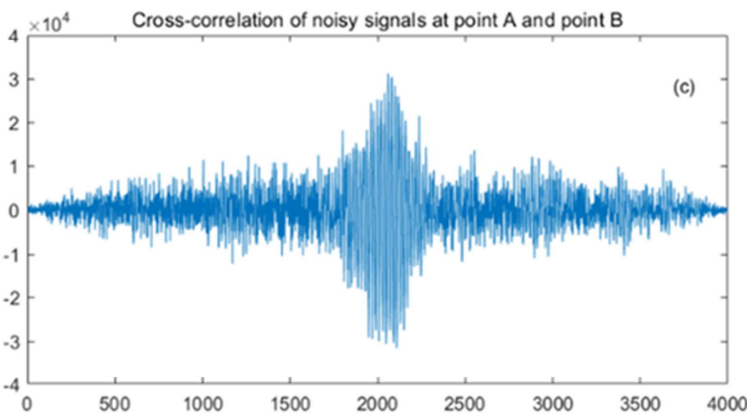

c. Cross-correlation of noisy signals at point $A$ and point $B$ function of the PD ultrasonic signal at point A before and after the CEEMDAN threshold denoising and the cross-correlation function of the ultrasonic signal at point $\mathrm{A}$ and $\mathrm{B}$ are obtained. Figure 7 shows the waveforms of the autocorrelation function and the cross-correlation function.

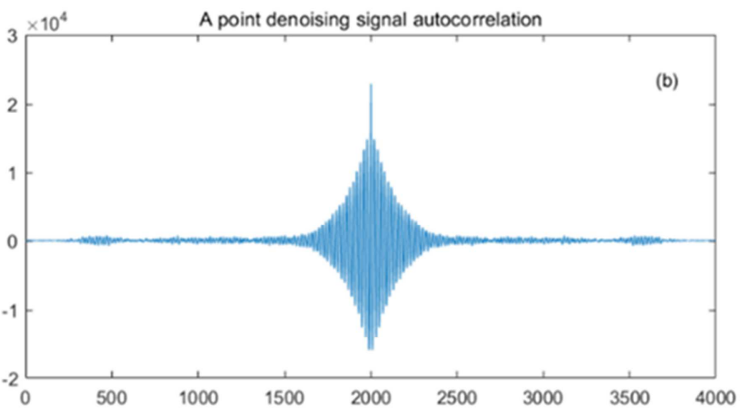

b. A point denoising signal autocorrelation

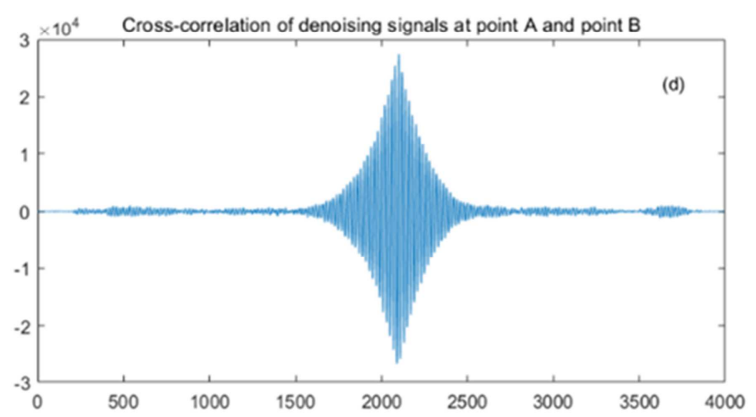

d. Cross-correlation of denoising signals at point $A$ and point $B$

Figure 7. Autocorrelation and cross-correlation waveform comparison chart.

It can be seen from the figure that the method described in the literature has been basically unusable in an environment with $\mathrm{SNR}=-20 \mathrm{~dB}$ [3]. The first main lobe of the correlation function after denoising is compared on the time axis multiple times, and the corresponding time difference estimation value is obtained, and the estimated value is subjected to histogram statistics, and the segment with the largest number of statistics is taken and averaged. The time difference is estimated to be
$9.98 \mu_{s}$. In order to verify the effectiveness of the proposed algorithm in low SNR environment, the generalized correlation method, LMS algorithm, the correlation coefficient waveform comparison method and the proposed algorithm are compared. All simulations are done 100 times. The average is taken as the final time difference estimate, and the signal-to-noise ratio is set to $10 \mathrm{~dB}$ to $-15 \mathrm{~dB}$.

Table 1. Estimation of time difference of different time difference estimation methods under different signal-to-noise ratios ( $\mu$ s ).

\begin{tabular}{|c|c|c|c|c|c|c|}
\hline SNR & $10 \mathrm{~dB}$ & $5 \mathrm{~dB}$ & Odb & $-5 d B$ & $-10 \mathrm{~dB}$ & $-15 d B$ \\
\hline Generalized correlation method & 9.992 & 9.956 & 9.924 & 9.244 & Invalid & Invalid \\
\hline LMS & 9.986 & 9.912 & 9.866 & 9.165 & Invalid & Invalid \\
\hline Correlation coefficient waveform comparison & 9.996 & 9.954 & 9.876 & 9.756 & 9.368 & 9.078 \\
\hline
\end{tabular}

It can be seen from Table 1 that as the signal-to-noise ratio drops to $-10 \mathrm{~dB}$, both the generalized correlation algorithm and the LMS algorithm have failed. The correlation coefficient waveform comparison method increases the error significantly after the signal-to-noise ratio drops below $-10 \mathrm{~dB}$. The proposed algorithm still maintains a good estimation accuracy in the environment with a signal-to-noise ratio of $-15 \mathrm{~dB}$.

\section{Conclusion}

In this paper, the CEEMDAN threshold denoising optimization correlation coefficient waveform comparison time difference estimation algorithm is proposed. For the characteristics that the PD ultrasonic signal is a narrowband signal, the correlation coefficient waveform comparison method is introduced to solve the generalized correlation 
method, LMS algorithm and other peak detection algorithms, which are commonly used for PD signal delay calculation, has low estimation accuracy. The power field environment is complex, and the PD signal is usually submerged in the noise. The CEEMDAN threshold denoising algorithm is introduced to improve the signal-to-noise ratio of the PD ultrasonic signal and improve the anti-noise performance of the algorithm.

By comparing the generalized correlation algorithm, LMS algorithm, correlation coefficient waveform comparison algorithm and the proposed algorithm for the time difference estimation of PD ultrasonic signals, The results show that in the low SNR environment, the accuracy of the first two algorithms is seriously reduced or even invalid, and the accuracy of the correlation waveform comparison method is also affected to a large extent. The waveform comparison algorithm of CEEMDAN threshold denoising optimization proposed in this paper still has high estimation accuracy for PD ultrasonic signals in low SNR environment, and has the advantage of being insensitive to bandwidth, which is beneficial to the subsequent positioning work.

\section{References}

[1] Jialin Liu, Ming Dong, Shan An. A Survey of Partial Discharge Detection and Location Technology of Power Transformers [J]. Insulation Materials, 2015 (08): 1-7.

[2] Fangcheng Lv, ying Zhang, han Wu. Research on Ultrasonic Localization Method Based on Improved RD_MUSIC Algorithm and Lean_Omitted Array [J]. Journal of North China Electric Power University (Natural Science Edition). 2018 (01): 1-6.

[3] Lei Yu, Shijie Wang, Peng Wang. Simulation and Experimental Study on Partial Discharge Ultrasonic Positioning of Transformer Windings [J]. Insulation Materials, 2019, 52 (6): 72-78.

[4] DANIELE S, SERGIO C. Adaptive time delay estimation using filter length constraints for source localization in reverberant acoustic environments [J]. IEEE Signal Processing Letters, 2013, 20 (5): 507-510.

[5] Wenhong LIU, Tianshuang QIU, Zhiyue LIN, et al. Estimation of propagation velocity of gastric electrical activity using LMSTDE [C] 7 VOLS, 2005-01-01: Institute of Electrical and Electronics Engineers Inc., 445 Hoes Lane / P. O. Box 1331, Piscataway, N, 2005: 5954-5957.

[6] LE B C, Yide WANG, VINCENT B, et al. Time delay and permittivity estimation by ground-penetrating radar with support vector regression [J]. IEEE Geoscience and Remote Sensing Letters, 2014, 11 (4): 873-877.
[7] Robert Hanus. Time delay estimation of random signals using cross-correlation with Hilbert Transform [J]. Measurement, 2019, 146.

[8] Wei XIA, Wenying JIANG, Lingfeng ZHU. An Adaptive Time Delay Estimator Based on ETDE Algorithm with Noisy Measurements [J]. Chinese Journal of Electronics, 2017, 26 (04): 760-767.

[9] Xiao Chen, Qu Zhenlin. Time Delay Estimation by Bispectrum Interpolation [J]. Sensors \& Transducers, 2013, 158 (11).

[10] Huijuan Wu, Yumei Wen, Jin Yang, Ping Li. Adaptive detection and location for water pipeline leaks in lower SNR and nonstationary environments [P]. Control and Automation, 2009. ICCA 2009. IEEE International Conference on, 2009.

[11] YANG GAO, Tianshuang QIU, Lan SHA, et al. Narrowband time delay estimation based on correlation coefficient [J]. Journal of Systems Engineering and Electronics, 2009 (05): 937- 941 .

[12] Lan Sha. Research on Time Delay Estimation Method for Narrowband Signals in Radio Passive Location [D] Dalian: Dalian University of Technology, 2009.

[13] Bo Sun, Jianwen Zhang, Leiluo Pan. Research on Partial Discharge Denoising Method Based on EMD. Insulation Materials, 2014 (03): 89-93.

[14] TORRES. A complete ensemble emprirical mode decomposition with adaptive noise [J]. IEEE Transaction on Signal Process, 2011 (2): 4144-4147.

[15] Chang Shu, Xiao Jin, ZiPin Li. CEEMDAN based distribution transformer discharge fault noise diagnosis method [J]. High voltage technology, 2018 (10): 2603-2611.

[16] Jianwen Zhang, Yang Liu, Dapeng Zhang. A new denoising method based on CEEMDAN and wavelet adaptive threshold $[\mathrm{J}]$. Electrical measurement and instrumentation, 2018 (10): $14-18+33$.

[17] Zhiman He, Jianwen Wang, Xinchuan Jiang. Research on calculation method of time delay of partial discharge UHF signal of switchgear based on EMD optimized bispectrum [J]. Smart power, 2018 (11): 59- 64.

[18] JiaCheng Gao, Yunqin Tian, Yongli Zhu. Wavelet packet denoising method based on partial set empirical mode decomposition and permutation entropy partial discharge signal [J]. Journal of Power Systems and Automation, 2018 (03): 1-7.

[19] Qi Liu. Research on Ultrasonic Testing Method for Partial Discharge of Power Transformer [D]. Shenyang: Shenyang University of Technology, 2017.

[20] Wenwen Zhao, Xingwen Zeng. A new EMD denoising method [J] Electronic technology, 2008 (05): 30-32+36. 